\title{
Streamlining the ethics review system
}

$\mathrm{Q}$ uebec and Ontario have made strides this year to increase the efficiency of multisite clinical trials by streamlining research ethics boards.

The streamlining process in Ontario is already showing positive results. Clinical Trials Ontario (CTO), an organization supported by the Ontario government, launched its web-based system to streamline applications and reviews in March and is seeing faster approval times.

"It's saving months off the time to get an ethics review, or at least weeks and weeks," said CTO President Susan Marlin.

Typically, gaining approval from an ethics board can take anywhere from six weeks to six months. For a multisite trial, that process is repeated at each site by a different board. That isn't the case, however, for reviews conducted by any of the 11 "CTO Qualified" research ethics boards.

"We can just do it once with one of our boards and it applies to all," said Marlin.

So far, CTO has reviewed 18 studies involving 45 research sites, with a median approval timeframe of 41 days, said Marlin. After a review board grants approval, an additional site can join the study in as little as five days.

In February, Quebec's Ministry of Health and Social Services launched a multicentre mechanism that also requires only one ethics review. The review process now has sites ready to go within six to eight weeks instead of six months.

Under the old system, redundant ethics approvals at additional research sites sometimes took so long that would-be study participants became anxious. Kristiana Salmon, a clinical research coordinator specializing in amyotrophic lateral sclerosis (ALS) at the Montréal Neurological Institute and Hospital, recalled a patient who travelled five hours to Montréal to see if she could enrol in a multicentre clinical trial. The patient lived much closer to a site for the trial in Que-

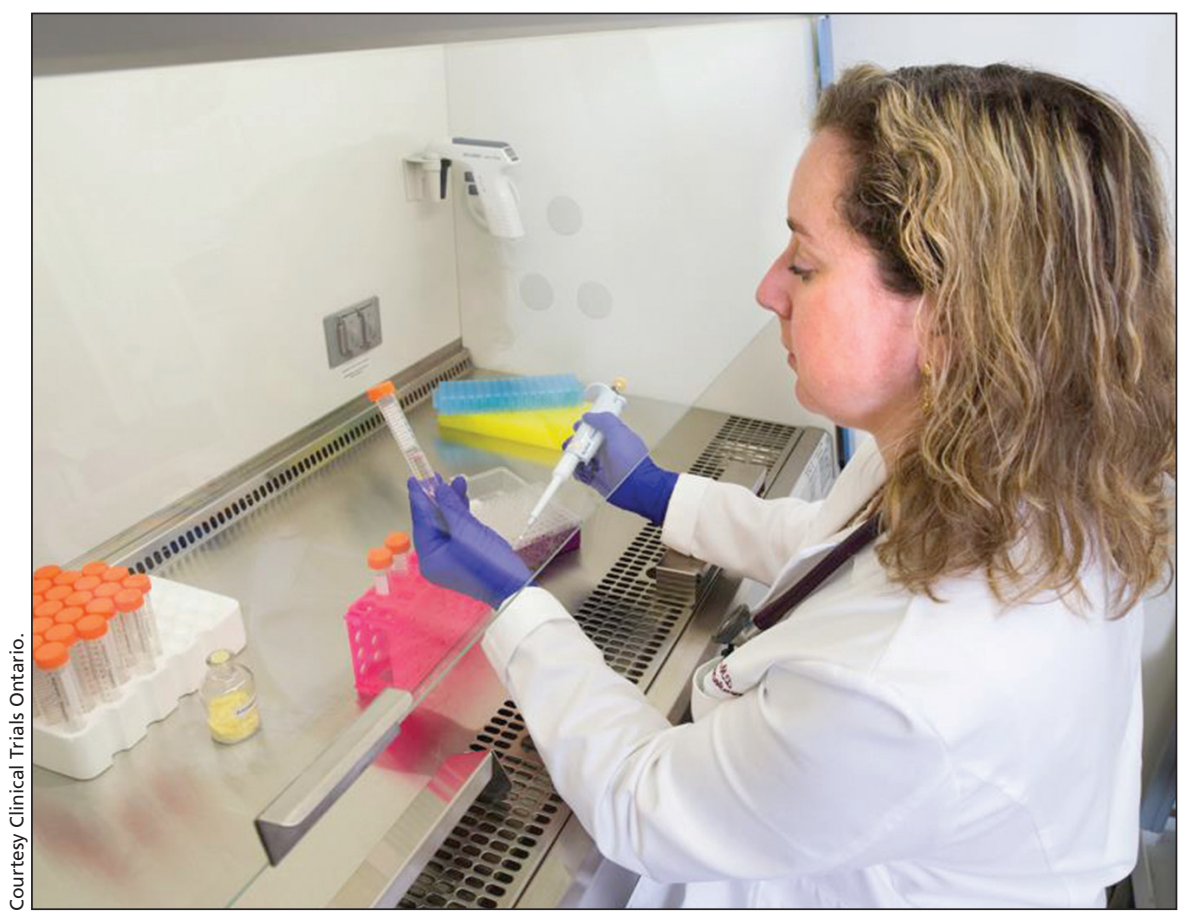

There are more than 3200 clinical trials underway in Ontario at any given time.

bec City, but that location was six months away from gaining ethics approval.

"These patients can't wait," said Salmon. "They can't wait six months for a trial to start. They'll fall out of eligibility."

Most clinical trial experts agree that the overall process for setting up multisite studies in Canada is often overly elaborate and time consuming, with protocols that vary by province and institution. For all trials, the first step is obtaining a no-objection letter from Health Canada, which has a guaranteed turnaround of 30 days. Then a research site seeks approval from its ethics board, a process that takes up to six months. The last step is contract negotiation between the sponsor of the trial and the institution running it, which can take from one to six months.

The Quebec health ministry is trying to simplify the whole process, said Franca Cantini, who is responsible for multicentre research at the Jewish General Hospital in Montréal. Many pharmaceutical companies go to Europe, China and India for their clinical trials, said Cantini - not only because it's cheaper but also to avoid the bureaucratic hoops they must jump through in Canada.

To stay competitive and timely the ministry also added "a formally mandated person" at each institution within the provincial health network to coordinate activities such as contract negotiations and resource evaluations by feasibility committees.

"It relieves the researcher and the research team from a lot of bureaucratic steps," said Cantini. "The ministry basically took it away from their responsibility and put the responsibility on the institution."

There is still a learning curve, said Cantini, and not everyone has embraced the system yet. "The tools are there but few people know about them and know how to use them." She estimated that it will take two to three years for the system to reach its full potential. - Shannon Lough, Ottawa, Ont.

CMAJ 2016. DOI:10.1503/cmaj.109-5199 